\title{
Gesundheitsreform auf Kosten der Prävention
}

W er in einer Zeitung blättert, den Briefkasten öffnet oder den Fernseher anschaltet, wird neben dem, was er eigentlich sucht, immer auch Beiträge einer anderen Art finden, in denen Kaufleute und Dienstleister etwas anzeigen, nämlich ihre Produkte oder Dienstleistungen. Obwohl uns vielleicht manchmal die geballte Ladung Werbung, die da so ständig auf uns herniedergeht, gehörig auf den Geist geht, so hält sie uns doch auch irgendwie auf dem Laufenden. Meist geht es nicht um die Information, dass es ein Produkt oder eine Dienstleistung gibt, sondern dass man „nur“ soundso viel dafür bezahlen muss. „Konkurrenz belebt das Geschäft“ heißt das Prinzip, und tatsächlich tut sich Kaufmann Meier schwer, uns seine Bananen für $5 €$ das Kilo zu verkaufen, wenn es Kaufmann Müller gelungen ist, uns darüber in Kenntnis zu setzen, dass er von uns nur die Hälfte will.

Lag diese Erfahrung aus dem täglichen Leben den Überlegungen des Sachverständigenrates des Bundesgesundheitsministeriums zu Grunde, gegen die ständig zunehmenden Kosten im Gesundheitswesen mit den Waffen der Marktwirtschaft zu Felde zu ziehen? „Mehr Wettbewerb ins System!“ riefen denn die verantwortlichen Gesundheitspolitiker wie einen Zauberspruch in die Mikrofone der Journalisten und machten eine Gesundheitsreform. Damit's nicht gleich so weh tut, hatten sie sich diesmal etwas Besonderes ausgedacht: eine Reform mit Etappen wie bei der Tour de France. Ohne dass es weiterer großer Ankündigungen und kontroverser Diskussionen bedarf, wird wohl mit Beginn des neuen Jahres eine weitere Etappe eingeläutet, eine wahre Königsetappe...

Ab 2009 sollen alle Mitglieder aller Gesetzlichen Krankenkassen den gleichen Beitragssatz bezahlen, in einen großen gemeinsamen Topf, den Gesundheitsfond. Die Idee dahinter ist, dass Krankenkassen dann nicht mehr mit einem niedrigen Beitragssatz um Mitglieder werben können, der ja nur durch einen besonders hohen Anteil gesunder (junger) Mitglieder oder durch eine angezogene Handbremse bei den Leistungen erreicht werden kann. Vielmehr haben dann (theoretisch) alle Krankenkassen die gleiche „Kriegskasse“ und wer damit für seine Mitglieder besonders attraktive Rahmenbedingungen gestaltet, hat die Nase vorn.

Die Sache hat allerdings einen Haken. Da manche Kassen besonders viele ältere und/ oder kränkere Mitglieder haben, funktioniert ein „fairer“ Wettbewerb nur, wenn dies bei der Weitergabe der Beiträge aus dem Gesundheitsfonds berücksichtigt wird. Morbiditätsassoziierter Risikostrukturausgleich, im Fachjargon „Morbi-RSA“ heißt das Instrument. Für die ca. 80 häufigsten bzw. teuersten Erkrankungen gibt es dann feste „Kopfprämien“ aus dem Gesundheitsfonds. Damit werden plötzlich kranke Mitglieder für die Krankenkasse ungleich interessanter als gesunde.

$\mathrm{Zu}$ Ende gedacht heißt das, wer sich für Prävention engagiert, also Geld ausgibt, dass seine Mitglieder gesund bleiben (oder die Krankheit möglichst nicht schlimmer wird), kriegt kein oder nur wenig Geld. Zu Ende gedacht heißt das auch, wäre die osteopathische Behandlung reguläre Kassenleistung, dann bekäme sie am Ende noch - erfolgsbedingt - Probleme... Da ist es schon fast ein Segen, wenn Therapeut und Patient sich ohne Rücksicht auf übergeordnete Interessen darauf verständigen können, dass eine gute Leistung einen guten Preis wert ist. Also „preiswert“ und damit konkurrenzfähig. Geht etwa am Ende „Wettbewerb“ auch ganz anders und sehr viel segensreicher für alle Beteiligten als es sich Sachverständige und Gesundheitspolitiker je träumen lassen?

\section{Die Herausgeber}

Titelbild: Oben: Neugeborenen-Sphenoid. Aufnahme: Prof. Dr. R. Breul, Anatomisches Institut der LMU, München. Unten: Sphenoid eines eineinhalbjährigen Kindes. Aus: Bardeleben, K von: Handbuch der Anatomie des Menschen. Erster Band: Skeletlehre. Jena: Fischer; 1896. Bearbeitung Pdesign, Stuttgart 2008. 